\title{
Correlation Between miR-125b Expression and Liver Fibrosis in Patients with Chronic Hepatitis C
}

\author{
Camelia Sultana ${ }^{1,2,{ }^{*}}$, Adelina Rosca ${ }^{1,2}$ and Simona Ruta ${ }^{1,2}$ \\ ${ }^{1}$ Carol Davila University of Medicine and Pharmacy, Bucharest, Romania \\ ${ }^{2}$ Emergent Disease Department, Stefan S. Nicolau Institute of Virology, Bucharest, Romania \\ "Corresponding author: Emergent Disease Department, Stefan S. Nicolau Institute of Virology, 285 Mihai Bravu Bvd, Bucharest, Romania. Tel/Fax:+40-213242590, Email: \\ camelia_sultana@yahoo.com
}

Received 2018 September 25; Revised 2018 November 24; Accepted 2018 December 11.

\begin{abstract}
Objectives: The study aimed to investigate the role of miR-125b as a non-invasive biomarker in chronic hepatitis C.

Methods: An observational study was conducted on 94 treatment-naïve HCV-infected patients (mean age $49.8 \pm 11.5$ years, $59.6 \%$ females). Liver fibrosis was assessed by transient elastography (TE) and the expression of miR-125b in plasma was quantified by real-time PCR.

Results: All patients were infected with HCV genotype $1 \mathrm{~b}$ and had active viral replication, $42.6 \%$ had significant cytolysis, and $73.4 \%$ had increased serum gamma-glutamyl transferase (GGT) values. Significant fibrosis (liver stiffness measured by TE of $>7.1 \mathrm{kPa}$ ) was present in $61.7 \%$ of the patients. No significant associations were found between miR-125b expression and baseline HCV viral load $(\mathrm{P}=0.56)$, IL28B polymorphisms $(\mathrm{P}=0.5)$, alpha-fetoprotein levels $(\mathrm{P}=0.27)$, and patients' gender $(\mathrm{P}=0.13)$ or age $(\mathrm{P}=0.5)$. In a univariate analysis, the miR-125b expression level was significantly correlated with ALT $(\mathrm{P}=0.001)$ and GGT levels $(\mathrm{P}<0.0001)$. An up-regulated expression of miR-125b was found in plasma samples from patients with advanced liver fibrosis as compared to those with mild/moderate fibrosis [mean miR-125b value $=0.002$ versus $0.001(\mathrm{P}=0.02)$ ]. In a multiple regression analysis, an upregulated miR-125b expression level remained independently in association only with significant fibrosis and increased GGT level $(\mathrm{P}=0.026$; $\mathrm{R}^{2}=0.242$ ).

Conclusions: An up-regulated miR-125b expression might be an indicator of severe liver fibrosis in patients with chronic hepatitis $\mathrm{C}$, independent of the viral replication level.
\end{abstract}

Keywords: miR-125b, Chronic Hepatitis C, Liver Fibrosis

\section{Background}

Chronic hepatitis $\mathrm{C}$ virus (HCV) infection affects $71 \mathrm{mil}-$ lion patients (1), remaining one of the most important public health problems worldwide. Although interferonfree combinations of direct-acting antivirals generate very high rates of sustained virologic response, patients with advanced fibrosis and cirrhosis remain at risk for the development of hepatocellular carcinoma (HCC), especially in the absence of fibrosis regression after successful therapy (2). Several epigenetic mechanisms, including noncoding microRNAs (miRNAs), are studied in order to understand the pathogenic mechanisms involved in the setting and progression of liver fibrosis and carcinogenesis (3). HCV infection modulates a series of cellular microRNAs, miR122 as a classic example, proving to be essential during HCV replicative cycle (4). Other miRNAs abundantly present in the liver, such as miR-125b as a highly conserved homolog of lin-4, are studied for their roles in major cellular pathways including inflammatory response, fibrogenesis, and hepatocellular oncogenesis $(5,6)$. The exosomal miR-125b level was correlated with the prognosis of hepatocellular carcinoma (7) and recently, plasma levels of miR-125b have been proposed as a non-invasive biomarker in chronic viral hepatitis (8), HBV-induced cirrhosis, and HBV-associated hepatocellular carcinoma (9).

\section{Objectives}

The present study aimed to investigate the potential value of miR-125b as a non-invasive biomarker in chronic hepatitis $\mathrm{C}$ and to evaluate the correlation between miR$125 \mathrm{~b}$ expression and viral or host factors involved in the progression of liver disease. 


\section{Methods}

An observational, retrospective study was conducted on 94 treatment-naïve HCV-infected patients [mean age $49.8 \pm 11.5$ years; range 30-68 years, $59.6 \%$ females]. The study was approved by the Ethics Committee of the Institute of Virology.

MiR-125b plasma expression was analyzed by realtime PCR with TaqMan ${ }^{\circledR}$ MicroRNA Assay (Applied Biosystems, USA), according to the manufacturer's protocol. Briefly, miRNA was isolated from $300 \mu \mathrm{L}$ of plasma using NucleoSpin ${ }^{\circledR}$ miRNA Plasma Kit (Macherey-Nagel, Germany). Reverse-transcription was performed using TaqMan ${ }^{\circledR}$ MicroRNA reverse transcription kit (Thermo Fisher Scientific, USA). The reverse-transcripted DNA fragments, resuspended in Hi-Di formamide, were amplified together with a DNA internal sizing standard in an ABI 7300 Genetic Analyzer. miRNA expression was normalized against those of an endogenous control: Cellular miR39 (Spike-In Cel-miR-39, Qiagen, USA) and against those of healthy volunteers, and determined by the $2^{-\Delta \Delta \mathrm{Ct}}$ method: $\Delta \mathrm{Ct}=\mathrm{Ct}$ miR-125b - Ct Cel-miR-39.

Serum HCV viral load was quantified using the RT-PCRCobas TaqMan HCV test, V2.0 (Roche Molecular Systems, Branchburg, NJ, USA), with a linear range of HCV-RNA between 15 and 100.000.000 IU/mL, and a lower limit of sensitivity of $15 \mathrm{IU} / \mathrm{mL}$.

Liver fibrosis was evaluated using a noninvasive method -transient elastography (TE, FibroScan, Echosens, France)- with good accuracy in differentiating mild/moderate fibrosis (corresponding to Metavir score F1/F2) from advanced fibrosis (F3/F4) at a cutoff of $7.1 \mathrm{kPa}$ for livers stiffness (10).

Polymorphism in the IL28B gene (SNP on chromosome 19-rs12979860) was tested with Custom TaqMan 5'-allelic discrimination assay (Applied Biosystems, USA).

The transferases levels were measured with the spectrophotometric standardized methods of the International Federation for Clinical Chemistry. The upper normal limit was $50 \mathrm{IU} / \mathrm{L}$ for Alanine Aminotransferase (ALT); for gamma-glutamyl transferase (GGT), it was $61 \mathrm{IU} / \mathrm{L}$ in men and $36 \mathrm{IU} / \mathrm{L}$ in women.

Serum alpha-fetoprotein (AFP) levels were measured using electrochemiluminescence (ECLIA, Roche Diagnostics), with normal values defined as less than $10 \mathrm{ng} / \mathrm{mL}$.

Statistical analysis was done using SPSS V.25 (Chicago, USA), employing Spearman rank correlation test for continuous variables and the Mann Whitney $U$ test for differences between various groups. A P value of less than 0.05 was considered statistically significant.

\section{Results}

All patients were infected with HCV genotype $1 \mathrm{~b}$ and had active viral replication (mean HCV viral load $=6.1 \pm$ $1.4 \log 10 \mathrm{IU} / \mathrm{mL}$ ). 55.3\% had high baseline HCV viral loads (above $5.8 \log 10 \mathrm{IU} / \mathrm{mL}$ ). The favorable CC IL28B polymorphism was present in only $21.3 \%$ of the patients, while $60.6 \%$ had the heterozygous CT genotype and the rest had the unfavorable TT polymorphism. Significant cytolysis, with serum ALT values 1.5 times greater than the upper limit of normal, was present in $42.6 \%$ of the patients; only had $27.7 \%$ normal ALT. Increased serum GGT values suggestive of cholestasis were present in $73.4 \%$ of the patients. Only had $11.7 \%$ of the patients high Alphafetoprotein serum values (AFP $>10 \mathrm{ng} / \mathrm{mL}$ ).

Advanced fibrosis, defined by a TE cutoff value of $\geq 7.1$ $\mathrm{kPa}$ for liver stiffness, was present in $61.7 \%$ of the patients, while the rest had mild/moderate fibrosis and none had cirrhosis.

In a univariate analysis, an up-regulated expression of miR-125b was found in plasma samples of patients with advanced liver fibrosis as compared to ones with mild/moderate fibrosis [mean miR-125b value $=0.002$ (range $0.0001-0.04$ ) versus 0.001 (range 0.0001-0.01) $(\mathrm{P}=$ $0.02)]$ and it was directly correlated with the degree of hepatic cytolysis (increased serum ALT values, $\mathrm{P}=0.001$ ) and cholestasis (increased GGT values, $\mathrm{P}<0.0001$ ), (Figure 1). No significant associations were found between miR-125b expression and other important viral and host predictors for liver disease evolution: High HCV viral load of $>5.8$ $\log 10 \mathrm{IU} / \mathrm{mL}(\mathrm{P}=0.56)$, increased alpha-fetoprotein levels $(\mathrm{P}=0.27)$, unfavourable IL28B genotype $(\mathrm{P}=0.5)$, patient's gender $(\mathrm{P}=0.13)$, or age $(\mathrm{P}=0.5)$.

In a multiple regression analysis, an upregulated miR125b expression remained independently associated only with advanced fibrosis and GGT levels, but not with serum $\operatorname{ALT}\left(\mathrm{P}=0.026 ; \mathrm{R}^{2}=0.242\right)$ (Table 1$)$.

\section{Discussion}

The present study reported a direct correlation between an up-regulated plasma miR-125b expression and advanced liver fibrosis in treatment-naïve HCV infected patients. This correlation was independent of the viral replication level and was not influenced by patients age, gender, or IL28 genotype as known predictors of the natural and on-treatment evolution of hepatitis C (11). It will be interesting to study the miR-125b expression in patients successfully treated with direct-acting antiviral regimens, as previous studies showed that miR-125b expression in peripheral blood mononuclear cells was significantly predictive of sustained virological response in interferon-treated 
A

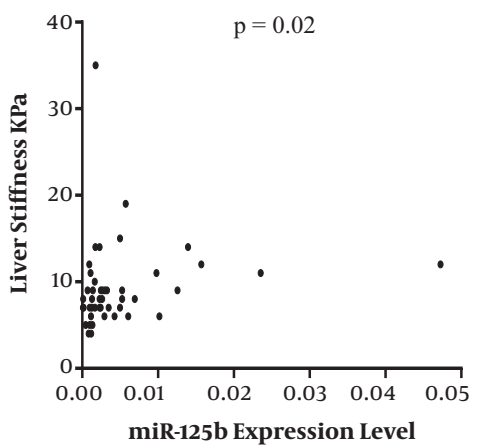

B

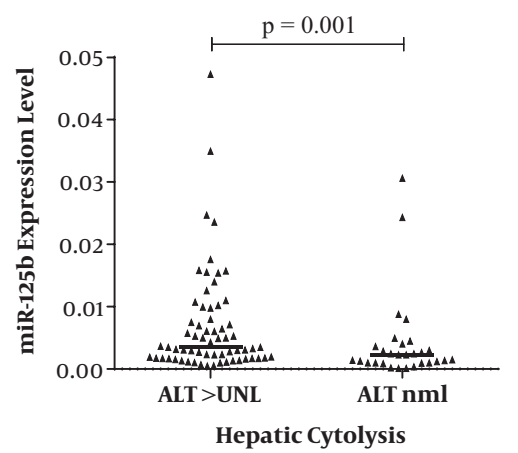

C

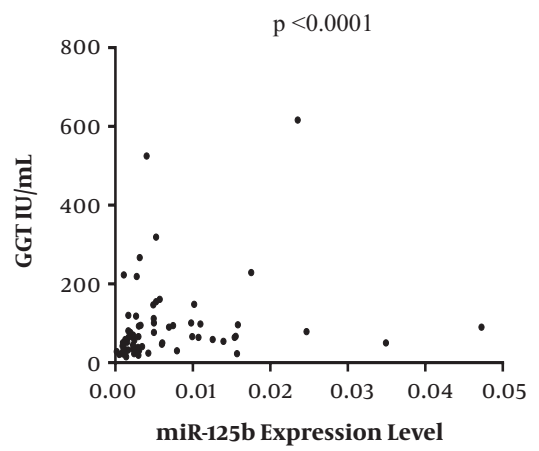

Figure 1. The relationship between miR-125b expression level and A, liver fibrosis; B, hepatocytolysis; and C, cholestasis

\begin{tabular}{lccc}
\hline Table 1. Multivariate Analysis of miRNA Expression. & & & \\
\hline Dependent Variable: miR125b & Std. Error & P & 95\% Confidence Interval \\
\hline Fibrosis & 0.002 & 0.03 & 0.0004 \\
Serum GGT & 0.00002 & 0.05 & -0.000005 \\
Serum ALT & 0.000004 & 0.49 & -0.00001 \\
\hline
\end{tabular}

HCV infected patients, again irrespective of interleukin28B genotype, age, or gender (12). The mechanisms by which miR-125b controls genes involved in fibrogenesis are still to be elucidated. In animal studies, this miRNA was found to be up-regulated in hepatic stellate cells, but not in hepatocytes; in vitro inhibition of miR-125b suppressed the expression of profibrogenic genes in primary hepatic stellate cells cultures (13). An abnormal in vitro expression of miR-125b is also associated with fibrosis in other organs, such as the heart, where this small transcript acts as a potent repressor of apelin and other antifibrotic mechanisms (14). Other studies have also reported increased serum levels of miR-125b in chronic HCV infected patients compared to normal controls, together with miR$146 \mathrm{a}$ and miR-155 that mediate a pro-inflammatory phenotype of monocytes, triggering TNF $\alpha$ production and maintaining a chronic immune activation, as the characteristics of chronic hepatitis C (15).

Previous reports identified specific miRNAs signatures for the diagnosis of hepatocellular carcinoma and proposed miR-125b expression as a predictor for liver cancer development or survival in $\operatorname{HCC}(3,5,16)$. We tested patients with chronic hepatitis $\mathrm{C}$, without markers of HCC progression, and identified a direct association between miR-125b up-regulation and advanced stages of liver fibrosis. In liver cancer, miR-125b down-regulation modulates several targets (BCL2, Bcl-W, and interleukin-6 receptor) to interfere with the apoptotic mechanisms and other targets (PIGF,
LIN28B) to regulate metastatic invasion and angiogenesis. Intriguingly, miR-125b up-regulation in some tumors indicates the oncogenic potential, while down-regulation in other tumors seems to indicate a tumor suppressive effect (17).

\subsection{Conclusions}

We suggest that up-regulated miR-125b expression is an indicator of severe liver fibrosis in patients with chronic hepatitis $\mathrm{C}$ and might serve as a novel prognostic biomarker, independent of the viral replication. Further studies regarding the longitudinal expression of miR-125b in treated patients are necessary to establish its value for the regression of fibrosis and its potential for therapeutic inhibition.

\section{Acknowledgments}

This paper was partially funded by PN-II-PT-PCCA-20113.2. programme grant No., 88/2012. The authors acknowledge the grant team for their support.

\section{Footnotes}

Conflicts of Interests: None declared. 
Ethical Considerations: We declare that the study complies with current ethical considerations, informed consent was obtained from all patients, and the study was approved by the Bioethics Committee of the Stefan S. Nicolau Institute of Virology.

Funding/Support: This paper was partially funded by PNII-PT-PCCA-2011- 3.2. programme grant No., 88/2012.

\section{References}

1. European Union HCV Collaborators. Hepatitis $C$ virus prevalence and level of intervention required to achieve the WHO targets for elimination in the European Union by 2030: A modelling study. Lancet Gastroenterol Hepatol. 2017;2(5):325-36. doi: 10.1016/S2468-1253(17)300456. [PubMed: 28397696].

2. Motoyama H, Tamori A, Kubo S, Uchida-Kobayashi S, Takemura $S$, Tanaka S, et al. Stagnation of histopathological improvement is a predictor of hepatocellular carcinoma development after hepatitis C virus eradication. PLoS One. 2018;13(3). e0194163. doi: 10.1371/journal.pone.0194163. [PubMed: 29534101]. [PubMed Central: PMC5849339].

3. Zhu Z, Zhang X, Wang G, Zheng H. Role of MicroRNAs in hepatocellular carcinoma. Hepat Mon. 2014;14(8). e18672. doi: 10.5812/hepatmon.18672. [PubMed: 25337143]. [PubMed Central: PMC4199151].

4. Roberts AP, Lewis AP, Jopling CL. miR-122 activates hepatitis C virus translation by a specialized mechanism requiring particular RNA components. Nucleic Acids Res. 2011;39(17):7716-29. doi: 10.1093/nar/gkr426. [PubMed: 21653556]. [PubMed Central: PMC3177192].

5. Kim JK, Noh JH, Jung KH, Eun JW, Bae HJ, Kim MG, et al. Sirtuin7 oncogenic potential in human hepatocellular carcinoma and its regulation by the tumor suppressors miR-125a-5p and miR-125b. Hepatology. 2013;57(3):1055-67. doi: 10.1002/hep.26101. [PubMed: 23079745].

6. Shrivastava S, Steele R, Ray R, Ray RB. MicroRNAs: Role in hepatitis C virus pathogenesis. Genes Dis. 2015;2(1):35-45. doi: 10.1016/j.gendis.2015.01.001. [PubMed: 25984557]. [PubMed Central: PMC4431773].

7. Liu W, Hu J, Zhou K, Chen F, Wang Z, Liao B, et al. Serum exosomal miR-125b is a novel prognostic marker for hepatocellular carcinoma. Onco Targets Ther. 2017;10:3843-51. doi: 10.2147/OTT.S140062. [PubMed: 28814883]. [PubMed Central: PMC5546809].
8. Akamatsu S, Hayes CN, Tsuge M, Miki D, Akiyama R, Abe H, et al. Differences in serum microRNA profiles in hepatitis $B$ and $C$ virus infection. J Infect. 2015;70(3):273-87. doi: 10.1016/j.jinf.2014.10.017. [PubMed: 25452043].

9. Giray BG, Emekdas G, Tezcan S, Ulger M, Serin MS, Sezgin O, et al. Profiles of serum microRNAs; miR-125b-5p and miR223-3p serve as novel biomarkers for HBV-positive hepatocellular carcinoma. Mol Biol Rep. 2014;41(7):4513-9. doi: 10.1007/s11033-014-3322-3. [PubMed: 24595450].

10. Castera L, Vergniol J, Foucher J, Le Bail B, Chanteloup E, Haaser M, et al. Prospective comparison of transient elastography, Fibrotest, APRI, and liver biopsy for the assessment of fibrosis in chronic hepatitis $C$. Gastroenterology. 2005;128(2):343-50. [PubMed: 15685546].

11. Zaltron S, Spinetti A, Biasi L, Baiguera C, Castelli F. Chronic HCV infection: Epidemiological and clinical relevance. BMC Infect Dis. 2012;12 Suppl 2. S2. doi: 10.1186/1471-2334-12-S2-S2. [PubMed: 23173556]. [PubMed Central: PMC3495628].

12. Hsi E, Huang CF, Dai CY, Juo SH, Chou WW, Huang JF, et al. Peripheral blood mononuclear cells microRNA predicts treatment outcome of hepatitis C virus genotype 1 infection. Antiviral Res. 2014;105:135-42. doi: 10.1016/j.antiviral.2014.03.003. [PubMed: 24637254].

13. You K, Li SY, Gong J, Fang JH, Zhang C, Zhang M, et al. MicroRNA125b promotes hepatic stellate cell activation and liver fibrosis by activating RhoA signaling. Mol Ther Nucleic Acids. 2018;12:57-66. doi: 10.1016/j.omtn.2018.04.016. [PubMed: 30195793]. [PubMed Central: PMC6023793].

14. Nagpal V, Rai R, Place AT, Murphy SB, Verma SK, Ghosh AK, et al. MiR125b is critical for fibroblast-to-myofibroblast transition and cardiac fibrosis. Circulation. 2016;133(3):291-301. doi: 10.1161/CIRCULATIONAHA.115.018174. [PubMed: 26585673]. [PubMed Central: PMC5446084].

15. Bala S, Tilahun Y, Taha O, Alao H, Kodys K, Catalano D, et al. Increased microRNA-155 expression in the serum and peripheral monocytes in chronic HCV infection. J Transl Med. 2012;10:151. doi: 10.1186/1479-587610-151. [PubMed: 22846613]. [PubMed Central: PMC3477071].

16. Fiorino S, Bacchi-Reggiani ML, Visani M, Acquaviva G, Fornelli A, Masetti M, et al. MicroRNAs as possible biomarkers for diagnosis and prognosis of hepatitis B- and C-related-hepatocellularcarcinoma. World J Gastroenterol. 2016;22(15):3907-36. doi: 10.3748/wjg.v22.115.3907. [PubMed: 27099435]. [PubMed Central: PMC4823242].

17. Banzhaf-Strathmann J, Edbauer D. Good guy or bad guy: The opposing roles of microRNA 125b in cancer. Cell Commun Signal. 2014;12:30. doi: 10.1186/1478-811X-12-30. [PubMed: 24774301]. [PubMed Central: PMC4011766]. 